bioRxiv preprint doi: https//doi.org/10.1101/2021.03 01.432277· this version posted March 1, 2021. The copyright holder for this preprint (which was not certified by peer review) is the author/funder, who has granted bioRxiv a license to display the preprint in perpetuity. It is made available under aCC-BY-NC-ND 4.0 International license.

\title{
A drastic shift in the energetic landscape of toothed whale sperm cells
}

L. Q. Alves ${ }^{1 \dagger}$, R. Ruivo ${ }^{1 \dagger}$, R. Valente ${ }^{1,2}$, M. M. Fonseca ${ }^{1}$, A. M. Machado ${ }^{1,2}$, S. Plön ${ }^{3}$, N. Monteiro ${ }^{2,4}$, D. García-Parraga ${ }^{5}$, S. Ruiz-Díaz ${ }^{6,7}$, M.L. Sánchez-Calabuig ${ }^{6,8}$, A. Gutiérrez-Adán $^{6 *}$, L. Filipe C. Castro ${ }^{1,2 *}$.

${ }^{1}$ CIMAR/CIIMAR - Interdisciplinary Centre of Marine and Environmental Research, University of Porto, Avenida General Norton de Matos, S/N, 4450-208 Matosinhos, Portugal.

${ }^{2}$ FCUP - Department of Biology, Faculty of Sciences, University of Porto (U. Porto), Rua do Campo Alegre, Porto, Portugal.

${ }^{3}$ Bayworld Centre for Research and Education (BCRE), Port Elizabeth, South Africa. Present address: Hanse Wissenschaftskolleg, Institute for Advanced Study, 27753

Delmenhorst, Germany

${ }^{4} \mathrm{CIBIO}$ - Research Centre in Biodiversity and Genetic Resources, Campus Agrário de Vairão, Rua Padre Armando Quintas, 4485-661 Vairão, Portugal

${ }^{5}$ Veterinary Services, L'Oceanográfic, Ciudad de las Artes y las Ciencias, Junta de Murs i Vals, s/n, 46013

${ }^{6}$ Departamento de Reproducción Animal, INIA, Av. Puerta de Hierro, 18, 28040 Madrid, Spain.

${ }^{7}$ Mistral Fertility Clinics S.L., Clínica Tambre, 28002 Madrid, Spain

${ }^{8}$ Department of Animal Medicine and Surgery, Faculty of Veterinary Science, University Complutense of Madrid, 28040, Madrid, Spain

*Correspondence to: filipe.castro@ciimar.up.pt, +351 223401800, ORCID ID: 00000001-7697-386X; agutierr@inia.es, +34 913473768, ORCID ID: 0000-0001-9893-9179

$\uparrow$ Equal Contribution

L. Q. Alves: luis.alves@ciimar.up.pt, +351 223401831, R. Ruivo: rruivo@ciimar.up.pt, +351 223401818, R. Valente: up201206792@fc.up.pt, +351 223401800, M. M.

Fonseca: mig.m.fonseca@gmail.com, +351 223401800, A. M. Machado: andre.machado@ciimar.up.pt, +351223401831, S. Plön: stephanie.ploen@gmail.com, +27-(0)76 3791067, +49-(0)4221 9160223, N. Monteiro: nuno.monteiro@cibio.up.pt, +351 252 660411, D. García-Parraga: dgarcia@oceanographic.org, +34 960470647, S. Ruiz-Díaz: sara.rd.1992@,gmail.com, +34 913473768, M.L. Sánchez-Calabuig: msanch26@ucm.es, +34913473765 
38 Abstract: Mammalia spermatozoa are a notable example of energetic

39 compartmentalization. While mitochondrial oxidative phosphorylation is restricted to the

40 midpiece, sperm-specific glycolysis operates in the flagellum. Consequently, these highly

41 specialized cells exhibit a clear adaptability to fuel substrates. This plasticity is essential

42 to ensure sperm motility, and is known to vary among species. Here we describe an

43 extreme example of spermatozoa-energetics adaptation. We show that toothed whales

44 exhibit impaired sperm glycolysis, due to gene and exon erosion, and demonstrate that

45 dolphin spermatozoa motility depends uniquely on endogenous fatty acid $\beta$-oxidation,

46 but not carbohydrates. Our findings substantiate the observation of large mitochondria in

47 spermatozoa, possibly boosting ATP production from endogenous fatty acids. This

48 unique energetic rewiring emphasizes the physiological body reorganisation imposed by

49 the carbohydrate-depleted marine environment.

50 Main Text: The rise of modern human societies from ancient civilizations implied a

51 division of labor and specialization: critical leverages towards the evolution of complex

52 interactions. Such trend is also noticeable in the evolution of biological complexity,

53 associated with the emergence of structured compartments and resource distribution

54 towards specific functions (e.g. multicellularity). At the cellular level, we can also recognize structural partitions. Yet, the compartmentalization of metabolic pathways, in time and space, within such structures is less intuitive (1). Mammalian spermatozoa

57 provide an illustrative example. The energy in the form of ATP production, vital for 58 motility, capacitation, and fertilization, is subcellularly separated in sperm cells. While

59 glycolysis provides a local, rapid, and low-yielding input of ATP along the flagellum 60 fibrous sheath, oxidative phosphorylation (OXPHOS), far more efficient over a longer

61 time frame, is concentrated in the midpiece mitochondria (2). The relative weight of 62 glycolysis and OXPHOS pathways in sperm function is species-dependent, and sensitive 
63 to oxygen and substrate availability (i.e., glucose, fructose, fatty acids, lactate, glycerol,

64 ketone bodies, or pyruvate) (3). For example, while OXPHOS was suggested to be

65 predominant in bull spermatozoa, in humans and mice, glycolysis is essential, notably in

66 the distal tail $(4,5)$. Besides partitioning energy production, sperm cell energetics display

67 an additional singularity: the occurrence of sperm-specific gene duplicates and alternative

68 spliced variants, although with conserved function (Fig. S1) (6).

69 The wider selective forces driving the compartmentalization and adaptability of this

70 energy system in mammalian species remain largely unknown; much like the impact of

71 ecosystem resource availability (e.g. carbohydrates, fatty acids, proteins) and dietary

72 adaptations in reproductive physiology traits (7). Here, we investigated the Cetacea, an

73 iconic group of fully aquatic and carnivorous marine mammals, evolutionarily related to

74 extant terrestrial herbivores. In this lineage, episodes of profound trait adaptation have

75 been accompanied by clear genomic signatures (8-10). To investigate the evolution of

76 sperm energetics, we started by analyzing the glycolysis rate-limiting enzyme,

77 glyceraldehyde-3-phosphate dehydrogenase, spermatogenic (Gapdhs) (11), in

78 mammalian genomes. Using a pseudogene inference pipeline, Pseudochecker (12), we

79 scrutinized the coding condition of Gapdhs in 163 genomes and found that this gene

80 displays a Pseudoindex, a probability measure of pseudogenization (functional

81 inactivation) (12), compatible with gene lesion events in an extremely restricted number

82 of mammalian lineages (Table S1; table S2) (13). 
bioRxiv preprint doi: https://doi.org/10.1101/2021.03 01.432277; this version posted March 1, 2021. The copyright holder for this preprint (which was not certified by peer review) is the author/funder, who has granted bioRxiv a license to display the preprint in perpetuity. It is made available under aCC-BY-NC-ND 4.0 International license.

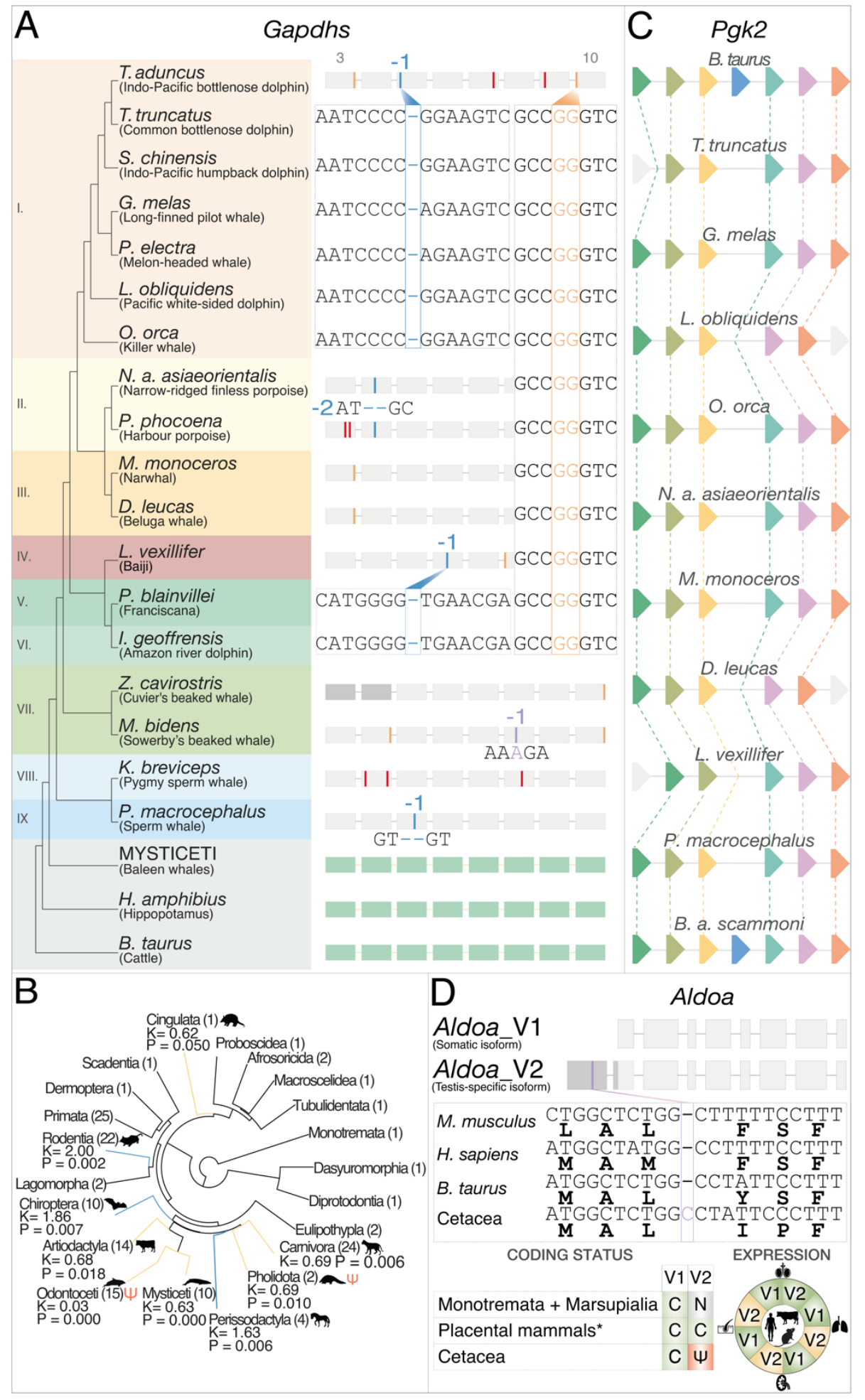

84 Fig. 1. Erosion of sperm-specific glycolysis genes. (A) The mutational landscape of 85 Gapdhs in Cetacea and hippopotamus (H. amphibius). Examples of the detected gene 86 disruptive mutations (insertions in purple, deletions in blue, in-frame premature stop 87 codons in red, and splice site mutations in yellow) are represented according to their 88 position within the affected exons (numerated on top, from the 3rd to the 10th exon - full 89 representation available in fig. S4). Odontoceti families are represented as follows: I. 90 Delphinidae, II. Phocoenidae, III. Monodontidae, IV. Lipotidae, V. Pontoporiidae, VI. 
91 Iniidae, VII. Ziphiidae, VIII. Kogiidae, IX. Physeteridae. (B) Branch-specific relaxation

92 parameters inferred for Gapdhs in mammals. (C) Comparative synteny maps of the Pgk2

93 genomic locus in Cetacea and Bos taurus (cow). Orthologous genes are joined by lines

94 (fig. S28). (D) Aldoa_v2 exon erosion in Cetacea. C-Coding, N-Not found, $\Psi$ -

95 Pseudogenized, Green-Expressed, Yellow-Not expressed. Silhouette images were

96 retrieved from http://phylopic.org/.

97

98 In effect, this pattern is almost exclusively limited to toothed whales (Odontoceti,

99 Cetacea). Next, we employed manual validation (Fig. S2) to inspect the coding sequence

100 of Gapdhs in species with a Pseudoindex higher than 2, including 18 species of toothed

101 whales (13), unveiling numerous gene-inactivating mutations in Odontoceti species (Fig.

102 1A; fig. S3-S21). Further examination of a bottlenose dolphin (Tursiops truncatus) sperm

103 transcriptome showed expression of Gapdhs with an aberrant splicing pattern, with

104 sequence reads encompassing the identified genome mutations (13) (fig. S22). A single

105 mutation is shared across all the examined Odontoceti species, with exception of the early

106 diverging beaked (Kogiidae) and sperm (Physeteridae) whales, which display

107 independent inactivating mutations (Fig. 1A). Gapdhs ORF-disrupting mutations were

108 also identified in other mammals, but were only found to be valid disrupting mutations in

109 the naked mole-rat, known to have simplified and degenerate sperm (14), and Pholidota

110 (pangolins) (fig. S4, S23-27). In addition, we identified relaxed selection in the whole

111 Cetartiodactyla clade, including baleen whales, Pholidota, Carnivora, and Cingulata

112 clades (Fig. 1B; table S3). Since we could not exclude the possibility of a functional

113 substitution by the somatic paralogue of Gapdhs (Gapdh), we next investigated Pgk2, a

114 glycolytic isozyme expressed uniquely during spermatogenesis and responsible for the

115 first ATP-generating step in this pathway (Fig. S1) (15). Despite overall synteny

116 conservation of the genomic locus in cow, hippopotamus, and baleen whales, together

117 with no significant selection relaxation or intensification (Table S3), we found that $P g k 2$

118 is sequence-deleted in all examined toothed whales (Fig. 1C; fig. S28-29). Again, Pgk2 
119 inactivating mutations were also deduced in pangolins and the naked mole rat (Fig. S29-

120 32). Out of the ten enzymes responsible for the conversion of glucose to pyruvate in sperm

121 cells (Fig. S1), we established that the sperm-cell restricted genes Gapdhs and Pgk2

122 display severe sequence lesions in Odontoceti species. Importantly, besides sperm-

123 specific genes, other glycolytic isoenzymes arise from alternative splicing, securing an

124 independent regulation from somatic splice variants (16). That is the case for Aldolase A

125 (AldoA), responsible for the synthesis of glyceraldehyde-3-phosphate, and presenting a

126 testis-specific splice variant, Aldoa_v2, with a distinct N-terminus exon (ด). Thus, we

127 next examined the sequence integrity of this sperm-exonic variant (Fig. 1D). We first

128 established that Aldoa_v2 emerged in the ancestor of placental mammals and is expressed

129 uniquely in testis (Fig. 1D). Moreover, we deduced the presence of ORF-disrupting

130 mutations within the sperm-specific exon in all of the examined Cetacea species (Fig. 1D,

131 fig. S33-60). 


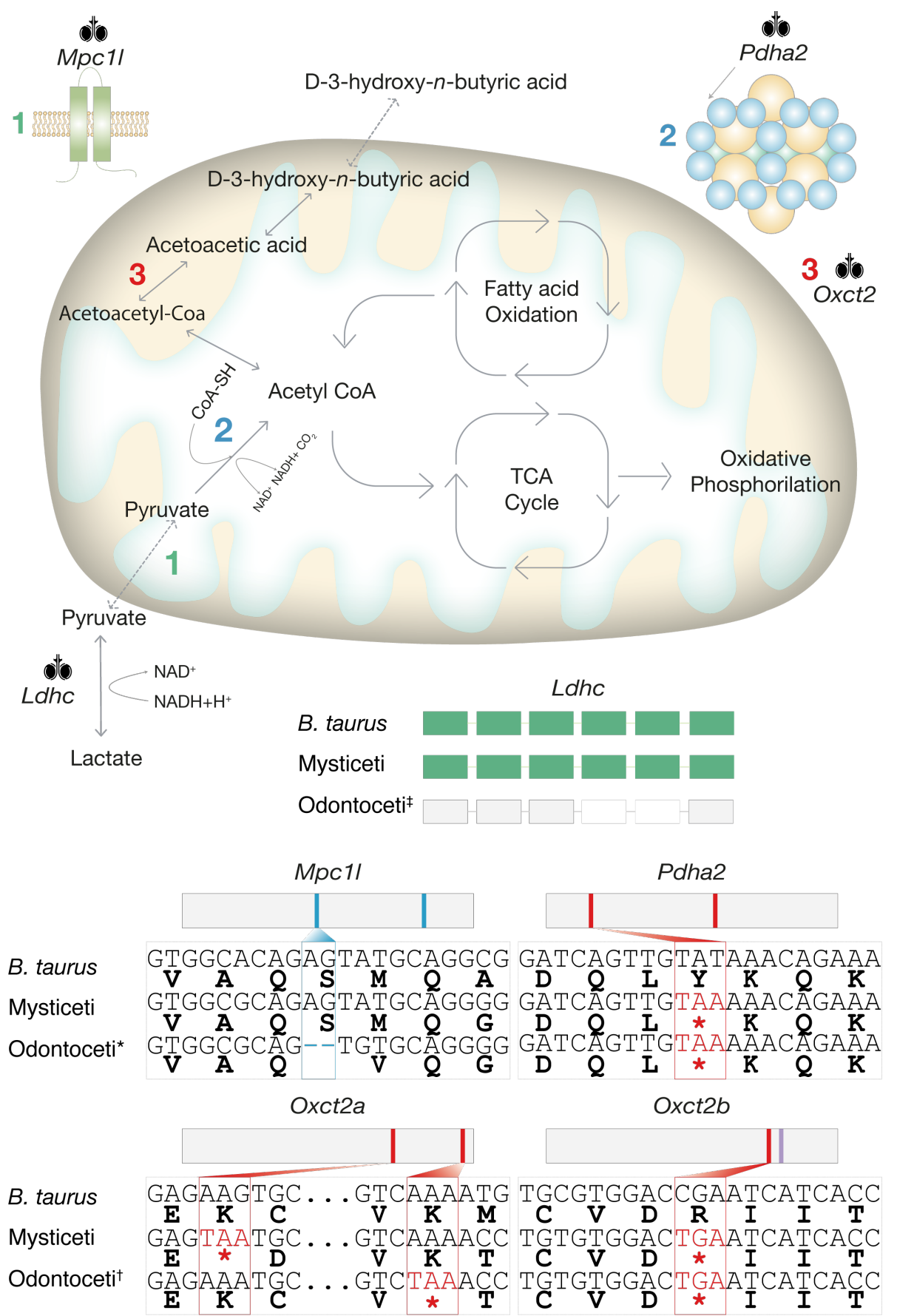

133 Fig. 2. Metabolic reactions and their sperm-specific genes participate in the 134 mitochondrial cellular respiration: oxidative decarboxylation of pyruvate, ketone 135 body catabolism, fatty acid $\beta$-oxidation, citric acid cycle, and oxidative 136 phosphorylation. ORF-disrupted genes in Cetacea are highlighted together with their 137 location in the pathway. Multiple sequence alignment of the coding sequences for Bos 138 taurus (cow), Mysticeti (baleen whales), and Odontoceti (toothed whales) emphasize the presence of disruptive mutations or exon loss ( $L d h c$, white rectangles). Nucleotide deletions are represented in blue, insertions in purple, and in-frame premature stop codons in red. *except I. geoffrensis (Amazon river dolphin), P. blainvillei (La Plata dolphin), Ziphiidae (beaked whales), P. macrocephalus (sperm whale), and K. breviceps (pigmy 
143 sperm whale). †except P. macrocephalus (sperm whale) for Oxct2a and O. orca (killer

144 whale) for $O x c t 2 b$.

146 A fully operational glycolysis pathway converts glycolysable sugars to pyruvate, with a

147 net gain of 2 ATPs. Sugar alcohols, such as sorbitol and glycerol can also be metabolized

148 entering the glycolytic pathway (5). The resulting pyruvate, at the intersection of

149 glycolysis and mitochondrial ATP production, can be (a) reversibly metabolized to

150 lactate, generating $\mathrm{NAD}^{+}$which can reintegrate the glycolytic pathway, sustaining additional ATP production (Fig. 2); or (b) transferred to the mitochondria, metabolized to acetyl-CoA and incorporated in the Krebs cycle and OXPHOS, generating up to 36 ATP molecules (Fig. 2). Additionally, acetyl-CoA may originate from glycolysis-independent sources such as fatty acid oxidation or ketone body catabolism. Together, most of these metabolic steps are controlled by spermatozoa-restricted gene orthologues, including

156 lactate dehydrogenase, $L d h c$, which oxidases NADH (17); a pyruvate specific transporter, Mpcll (18); a converter of pyruvate to acetyl-CoA performed by the pyruvate dehydrogenase complex composed of several genes, including the sperm restricted Pdha2 (19) (Fig. 2); glycerol kinase 2 (Gk2), which catalyzes the production of glycerol-3-

160 phosphate from glycerol, which can be enzymatically converted into glyceraldehyde-3-

161 phosphate (20) (Fig. 2); and, the 3-Oxoacid CoA-transferase 2 (Oxct2) which converts ketone body-derived acetoacetate into acetoacetyl-CoA in sperm cells (21) (Fig. 2). We examined each gene orthologue sequence in various mammal species and found numerous ORF-disrupting mutations or exon-loss events in most Cetacea species (Fig. 2;

165 fig. S61-S158). Overall, our results indicate the full dismantling of key energy-producing 166 pathways in Odontoceti species: including glycolysis, pyruvate and lactate production, mitochondrial pyruvate uptake and conversion to acetyl-CoA and mitochondrial ketone 
169 of ketone body synthesis in Cetacea (22). In contrast, Mysticeti exhibit sparse gene loss

170 events, with the inactivation of ketone body usage and pyruvate to acetyl-CoA

171 conversion, suggesting a metabolic dissociation between glycolysis and mitochondrial

172 pathways. Yet, in Mysticeti, glycolysis is most likely not fully abolished. The erosion of

173 Aldoa_v2 and retention of a functional Gapdhs suggest that partial glycolysis could occur

174 from non-sugar substrates with the production of the intermediary glyceraldehyde-3-

175 phosphate from glycerol; also, Aldoa_v2 could be functionally substituted by the somatic

176 paralogue. This landscape of gene erosion suggests a profound restructuring of energy

177 production in Odontoceti sperm cells, and to a lesser degree in Mysticeti. Together, these

178 findings raise the hypothesis of OXPHOS as the sole energy provider in sperm cells, a

179 physiological arrangement previously unknown in mammalian spermatozoa. Yet, a

180 critical question emerges: How do Odontoceti sperm cells obtain the required acetyl-CoA

181 to feed mitochondrial OXPHOS? A natural candidate is mitochondrial fatty acid $\beta$ -

182 oxidation.

183
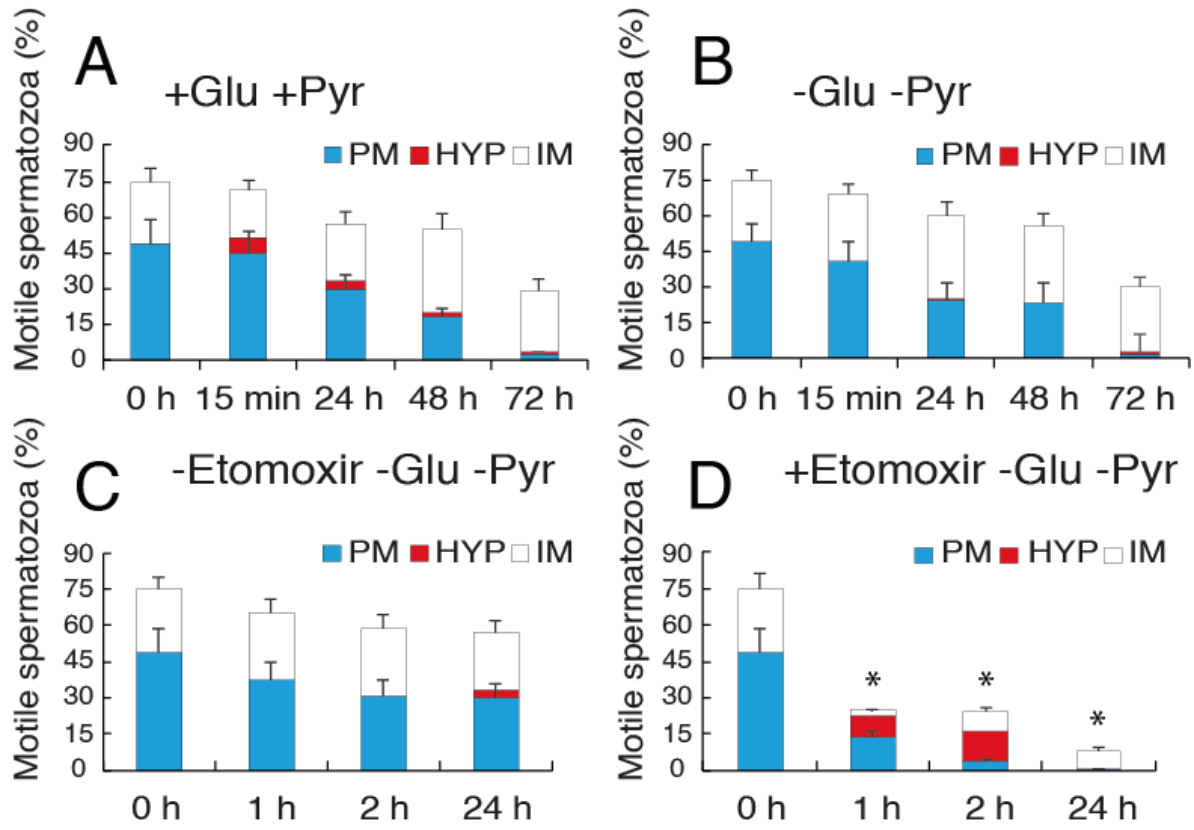
186 Fig. 3. Energetic substrate assays of dolphin sperm motility. Dolphin sperm samples

187 were incubated in the presence $(\mathbf{A})$ or absence $(\mathbf{B})$ of glucose and pyruvate. Total, 188 progressive (PM; Movie S1), hyperactive (HYP; Movie S2), and irregular (IM; Movie 189 S3) motility measured using the Integrated Semen Analysis System (ISAS) at 15 min, 24 $190 \mathrm{~h}, 48 \mathrm{~h}$, and $72 \mathrm{~h}$. No differences in total or progressive sperm motility were observed 191 between (A) and (C). Sperm samples were incubated in the absence (C) or presence (D) 192 of etomoxir; motility was measured using ISAS at $1 \mathrm{~h}, 2 \mathrm{~h}$, and $24 \mathrm{~h}$. Total and progressive 193 motility dropped significantly in the presence of etomoxir. Data are expressed as mean \pm 194 S.E. $* P<0.01$.

195

196 To experimentally validate this hypothesis, we examined dolphin sperm motility and movement longevity, an indicator of exhaustion of reserve energy sources, in the presence/absence of the glycolytic substrate glucose, the glycolysis end-product pyruvate, as well as etomoxir, a specific inhibitor of carnitine palmitoyl-transferase 1a (Cptla) and blocker of mitochondrial fatty acid $\beta$-oxidation (Fig. 3A-D) (23). Dolphin sperm cells maintained normal motility for more than 3 days, even without glucose and pyruvate supplementation (Fig. 3A and 3B), in clear contrast with mice and bull sperm cells, which typically stop moving or display reduced mobility shortly after 1 and 3 hours, respectively

(23). Either with or without supplementation, the percentage of cells exhibiting progressive or irregular motility was similar, with only a minute increase in hyperactivated motility when glucose and pyruvate were added to the incubation media

207 (Fig. 3A and 3B). In contrast, when exposed to etomoxir, cell motility dropped after only

2081 hour of incubation, significantly differing from the control [Control $1 \mathrm{~h}: 65.3 \% \pm 4.4 \%$

209 (mean \pm S.E.); etomoxir $1 \mathrm{~h}: 24.8 \% \pm 2.8 \%,(\mathrm{p}<0.01)]$, with progressive motility 210 practically disappearing after $2 \mathrm{~h}$ of incubation (Fig 3C and D). An increased number of 211 spermatozoa with hyperactive-like motility was also observed, when compared to the control group, at 1 and 2 hours of incubation with etomoxir (Fig 3D). This hyperactive-

213 like state is different from normal hyperactive motility (displaying an increased amplitude

214 of lateral head displacement and decreased straight-line and curvilinear velocities), but 
215 similar to that observed in subpopulations of bull sperm that die very quickly after

216 freezing/thawing (24). At 24 hours of incubation, only a small percentage of sperm cells

$217(9.3 \% \pm 1.4 \%)$ remained motile, displaying irregular motility patterns (Fig 3D).

218 Etomoxir-dependent $\beta$-oxidation inhibition was previously shown to decrease sperm

219 motility in various species including humans (25) or boar (26). Yet, in dolphin sperm,

220 such inhibition yielded faster and more pronounced effects on sperm motility, with

221 progressive motility diverted to hyperactive-like states, ultimately leading to irregular

222 sperm movement patterns. Overall, these findings showcase the complete dismantling of

223 the glycolytic pathway in sperm cells while support the central role of $\beta$-oxidation in ATP

224 generation to fuel normal sperm motility.
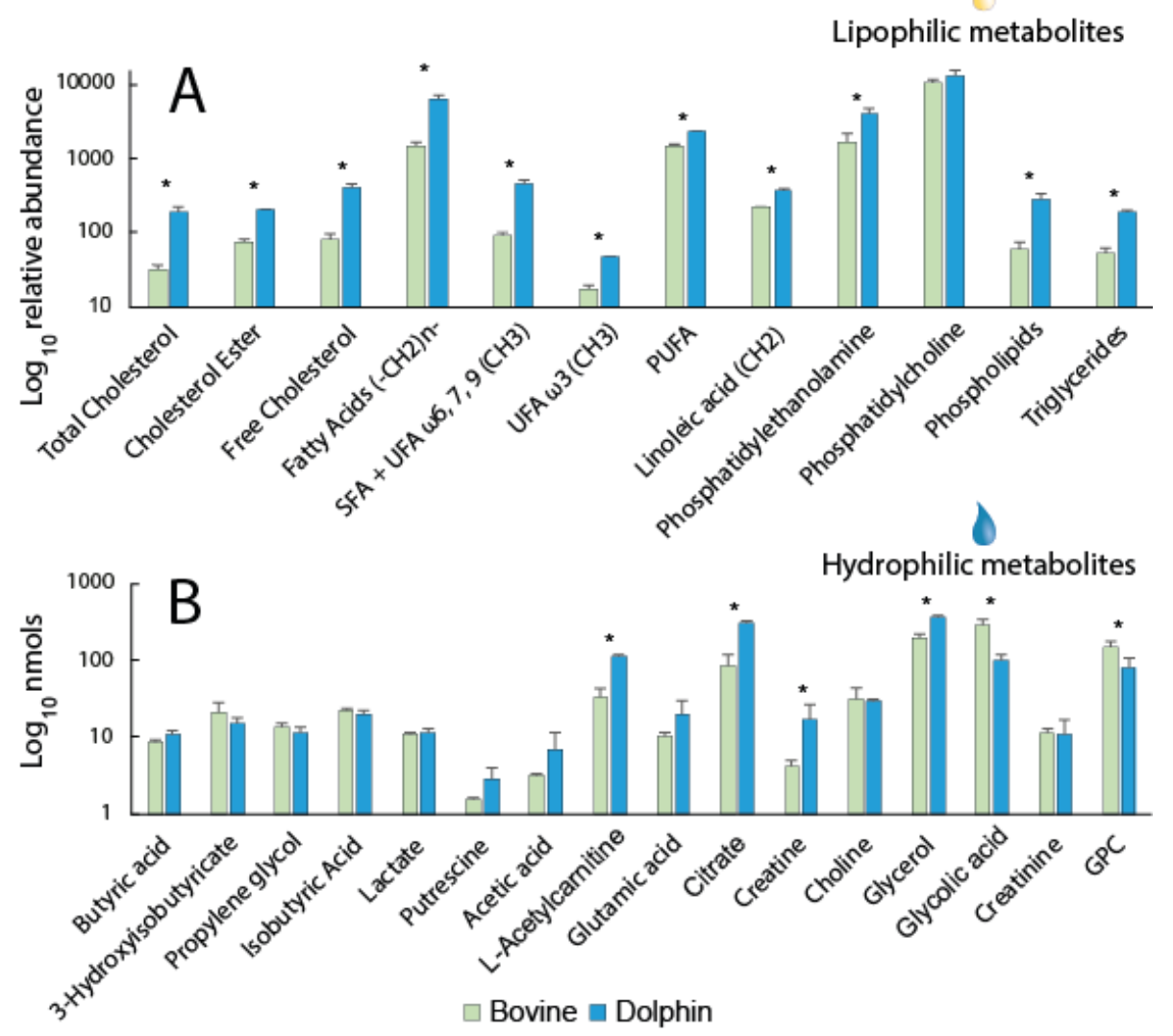

226 Fig. 4. Metabolites identified in bull and dolphin sperm extracts by proton nuclear magnetic resonance $\left({ }^{1} \mathrm{H}\right.$ NMR) spectroscopy using lipophilic (A) and hydrophilic (B) FA: fatty acid; SFA: saturated fatty acid; UFA: unsaturated fatty acid; PUFA: polyunsaturated fatty acid; GPC: glycerophosphocholine. 
231 To further identify the endogenous metabolites able to sustain the described cellular

232 behavior, we compared sperm from bottlenose dolphins and bull using proton nuclear

233 magnetic resonance ( ${ }^{1} \mathrm{H}$ NMR) spectroscopy. A total of 12 metabolites were identified in

234 the lipophilic phase and 16 metabolites in the hydrophilic phase (Fig. 4A and 4B). As

235 expected, fatty acid content, in the form of free fatty acids, triglycerides, or cholesterol

236 esters, was significantly higher in dolphin sperm, supporting the use of these lipophilic

237 compounds as substrates for mitochondrial energy production (Fig. 4A). Phospholipids,

238 notably phosphatidylethanolamine, were also more abundant in dolphin sperm, as well as

239 free cholesterol (Fig. 4A). In the hydrophilic phase, L-acetylcarnitine, citrate, creatine,

240 and glycerol content were higher in dolphin sperm than in bull (Fig. 4B). L-

241 acetylcarnitine is the acetylated and most common derivative of L-carnitine, required for

242 Cptla-dependent fatty acid shuttling into mitochondria for $\beta$-oxidation; citrate is an

243 intermediary of the Krebs cycle, resulting from the condensation of oxaloacetate and

244 acetyl-CoA, and a feedback inhibitor of glycolysis; creatine participates in the creatine-

245 phosphocreatine shuttle, an enzymatic energy transport system relying on

246 phosphocreatine diffusion from the mitochondria and subsequent regeneration of ATP

247 and creatine in the cytoplasm (27); and glycerol a major component of triglycerides and

248 phospholipids. Together these findings support a drastic modification of sperm energetics

249 in Odontoceti species towards fatty acid $\beta$-oxidation-fueled OXPHOS.

250 Unlike other mammals, the complete demolishing of glycolysis and shift towards

251 mitochondrial-enclosed metabolic pathways allowed the maintenance of normal sperm

252 functions in Odontoceti. For instance, the inactivation of glycolytic genes, in humans and

253 mice, induces defects in sperm structure and motility leading to infertility in humans and

254 mice (28-31) (Table S4); while in the eusocial naked mole-rat, with a dominant male

255 mating strategy, sperm is abnormal (14). Yet, in dolphins, sperm quality is generally very 
high, few abnormal sperm, in agreement with their promiscuous mating system based on sperm competition (32). This unique metabolic rearrangement was possibly accompanied by an increase in mitochondrial activity and/or ATP diffusion along the flagellum (i.e. creatine-phosphocreatine shuttle). Curiously, such metabolic burden on mitochondria is

260 paralleled by an also peculiar phenotype in Odontoceti when compared to other

261 Artiodactyla, including Mysticeti: bigger mitochondria with augmented cristae, resulting in larger midpiece volumes (32) (Fig S159 and S160).

263 The evolution of such a unique energetic system in Odontoceti sperm cells most probably emerged as a consequence of dietary adaptations and resource availability (33).

265 Odontoceti dietary intake, such as that of the common bottlenose dolphin, is characterized

266 by high-protein and low-carbohydrate content, leading to an overall low glycolytic activity (34). Glucose to fatty acid shifts in energy metabolism have also been described in Cetacea skeletal muscle as a result of gene inactivation (9). Surprisingly, circulating glucose levels in healthy dolphins are high (35). The fate of such high glucose stores is most likely the brain. Brain metabolic energy is exclusively provided by glucose or fatty acid-derived ketone bodies; yet, the synthesis of the latter is impaired in Cetacea (22).

272 Odontoceti, but not Mysticeti, have large brain-to-mass ratios and encephalization 273 quotients similar to non-human primates (36). Such critical concatenation of events likely

274 diverted the usage of glucose, a scarce resource in aquatic environments, from peripheral 275 functions in order to support the elevated energy demands of the brain (37). Additionally, 276 this energetic system, yielding good quality and enduring spermatozoa, is probably 277 favored in a polyandry system with sperm competition, such as that exhibited by many 278 Odontoceti species, and facilitates cell motility in complex vaginal folds (or 279 pseudocervices) hypothesized to restrict sperm access to the upper reproductive tract (38).

280 Our findings also have vital implications for captive reproduction programs across the 
bioRxiv preprint doi: https://doi.org/10.1101/2021.03.01.432277; this version posted March 1, 2021. The copyright holder for this preprint (which was not certified by peer review) is the author/funder, who has granted bioRxiv a license to display the preprint in perpetuity. It is made available under aCC-BY-NC-ND 4.0 International license.

world. Considering that dolphin sperm utilizes fatty acids as an energy source supporting

motility, the formulation of sperm storage media may need to be revisited.

\section{References}

1. A. Zecchin, P. C. Stapor, J. Goveia, P. Carmeliet, Metabolic pathway compartmentalization: an underappreciated opportunity? Curr. Opin. Biotechnol. 34, 73-81 (2015).

2. M. J. Freitas, S. Vijayaraghavan, M. Fardilha, Signaling mechanisms in mammalian sperm motility†. Biol. Reprod. 96, 2-12 (2017).

3. M. Tourmente, P. Villar-Moya, E. Rial, E. R. S. Roldan, Differences in ATP Generation Via Glycolysis and Oxidative Phosphorylation and Relationships with Sperm Motility in Mouse Species. J. Biol. Chem. 290, 20613-20626 (2015).

4. B. T. Storey, Mammalian sperm metabolism: oxygen and sugar, friend and foe. Int. J. Dev. Biol. 52, 427-437 (2008).

5. M. M. Misro, T. Ramya, in Male Infertility: Contemporary Clinical Approaches, Andrology, ART \& Antioxidants, S. J. Parekattil, A. Agarwal, Eds. (Springer New York, New York, NY, 2012), pp. 209-223.

6. S. A. Vemuganti et al., Three male germline-specific aldolase A isozymes are generated by alternative splicing and retrotransposition. Dev. Biol. 309, 18-31 (2007).

7. A. Ishikawa et al., A key metabolic gene for recurrent freshwater colonization and radiation in fishes. Science (New York, N.Y.) 364, 886 (2019).

8. W. K. Meyer et al., Ancient convergent losses of Paraoxonase 1 yield potential risks for modern marine mammals. Science (New York, N.Y.) 361, 591 (2018).

9. M. Huelsmann et al., Genes lost during the transition from land to water in cetaceans highlight genomic changes associated with aquatic adaptations. Sci. $A d v$. 5, eaaw6671 (2019).

10. M. R. McGowen et al., Positive Selection and Inactivation in the Vision and Hearing Genes of Cetaceans. Mol. Biol. Evol. 37, 2069-2083 (2020).

11. K. Miki et al., Glyceraldehyde 3-phosphate dehydrogenase-S, a sperm-specific glycolytic enzyme, is required for sperm motility and male fertility. Proc Natl Acad Sci U S A 101, 16501-16506 (2004).

12. L. Q. Alves et al., PseudoChecker: an integrated online platform for gene inactivation inference. Nucleic Acids Res. 48, W321-W331 (2020).

13. Further details are available in the supplementary materials.

14. G. van der Horst, L. Maree, S. H. Kotzé, M. J. O'Riain, Sperm structure and motility in the eusocial naked mole-rat, Heterocephalus glaber : a case of degenerative orthogenesis in the absence of sperm competition? BMC Evol. Biol. 11, 351 (2011).

15. P. V. Danshina et al., Phosphoglycerate Kinase 2 (PGK2) Is Essential for Sperm Function and Male Fertility in Mice1. Biol. Reprod. 82, 136-145 (2010).

16. N. Nakamura, C. Mori, E. M. Eddy, Molecular Complex of Three TestisSpecific Isozymes Associated with the Mouse Sperm Fibrous Sheath: Hexokinase 1, Phosphofructokinase M, and Glutathione S-Transferase mu class 51. Biol. Reprod. 82, 504-515 (2010). 
17. F. Odet et al., Expression of the Gene for Mouse Lactate Dehydrogenase C (Ldhc) Is Required for Male Fertility1. Biol. Reprod. 79, 26-34 (2008).

18. B. Vanderperre et al., MPC1-like Is a Placental Mammal-specific Mitochondrial Pyruvate Carrier Subunit Expressed in Postmeiotic Male Germ Cells. J. Biol. Chem. 291, 16448-16461 (2016).

19. V. Kumar, N. Rangaraj, S. Shivaji, Activity of Pyruvate Dehydrogenase A (PDHA) in Hamster Spermatozoa Correlates Positively with Hyperactivation and Is Associated with Sperm Capacitation1. Biol. Reprod. 75, 767-777 (2006).

20. K. Shimada, H. Kato, H. Miyata, M. Ikawa, Glycerol kinase 2 is essential for proper arrangement of crescent-like mitochondria to form the mitochondrial sheath during mouse spermatogenesis. J. Reprod. Dev. 65, 155-162 (2019).

21. H. Tanaka et al., Ketone bodies could support the motility but not the acrosome reaction of mouse sperm. Int. J. Androl. 27, 172-177 (2004).

22. D. Jebb, M. Hiller, Recurrent loss of HMGCS2 shows that ketogenesis is not essential for the evolution of large mammalian brains. eLife 7, e38906 (2018).

23. F. A. Navarrete et al., Transient Sperm Starvation Improves the Outcome of Assisted Reproductive Technologies. Front. Cell Dev. Biol. 7, (2019).

24. R. Muiño, C. Tamargo, C. O. Hidalgo, A. I. Peña, Identification of sperm subpopulations with defined motility characteristics in ejaculates from Holstein bulls: effects of cryopreservation and between-bull variation. Anim. Reprod. Sci. 109, 27-39 (2008).

25. A. Amaral et al., Human Sperm Tail Proteome Suggests New Endogenous Metabolic Pathways. Mol. Cell. Proteom. 12, 330-342 (2013).

26. Z. Zhu et al., Exogenous Oleic Acid and Palmitic Acid Improve Boar Sperm Motility via Enhancing Mitochondrial B-Oxidation for ATP Generation. Animals 10, (2020).

27. P. P. Asseo, D. K. Panidis, J. S. Papadimas, D. G. Ikkos, Creatine kinase in seminal plasma of infertile men: activity and isoenzymes. Int. J. Androl. 4, 431439 (1981).

28. X. Liu, Q. Li, W. Wang, F. Liu, Aberrant expression of sperm-specific glycolytic enzymes are associated with poor sperm quality. Mol. Med. Rep. 19, 2471-2478 (2019).

29. W. C. L. Ford, Glycolysis and sperm motility: does a spoonful of sugar help the flagellum go round? Hum. Reprod. Update 12, 269-274 (2006).

30. E. Goldberg, The Sperm Specific Form of Lactate Dehydrogenase (LDHC4) is Required for Fertility and is an Attractive Target for Male Contraception (A Review). Biol. Reprod., (2020).

31. D. Paoli et al., Sperm glyceraldehyde 3-phosphate dehydrogenase gene expression in asthenozoospermic spermatozoa. Asian J. Androl. 19, 409-413 (2017).

32. G. van der Horst, K. Medger, D. Steckler, I. Luther, P. Bartels, Bottlenose dolphin (Tursiops truncatus) sperm revisited: Motility, morphology and ultrastructure of fresh sperm of consecutive ejaculates. Anim. Reprod. Sci. 195, 309-320 (2018).

33. D. Derous, J. Sahu, A. Douglas, D. Lusseau, M. Wenzel, Comparative genomics of cetartiodactyla: energy metabolism underpins the transition to an aquatic lifestyle. Conserv. Physiol. 9, (2021).

34. M. Suzuki, M. Yoshioka, Y. Ohno, Y. Akune, Plasma metabolomic analysis in mature female common bottlenose dolphins: profiling the characteristics of 
metabolites after overnight fasting by comparison with data in beagle dogs. Sci. Rep. 8, 12030 (2018).

35. S. K. Venn-Watson, S. H. Ridgway, Big brains and blood glucose: common ground for diabetes mellitus in humans and healthy dolphins. Comp. Med. 57, 390-395 (2007).

36. A. M. Boddy et al., Comparative analysis of encephalization in mammals reveals relaxed constraints on anthropoid primate and cetacean brain scaling. $J$. Evol. Biol. 25, 981-994 (2012).

37. A. Krüger et al., Transcriptome analysis reveals a high aerobic capacity in the whale brain. Comp. Biochem. Physiol. A Mol. Integr. Physiol. 240, 110593 (2020).

38. D. N. Orbach, B. Hedrick, B. Würsig, S. L. Mesnick, P. L. R. Brennan, The evolution of genital shape variation in female cetaceans. Evolution 72, 261-273 (2018).

\section{Acknowledgments:}

This research was funded by COMPETE 2020, Portugal 2020, by the European Union through the ERDF, grant number 031342, by FCT through national funds (PTDC/CTAAMB/31342/2017), and by grant RTI2018-093548-BI00 from the Spanish Ministry of Science and Innovation. R.V. is funded by the FCT under the grant SFRH/BD/144786/2019. The study was also supported by Trainers and veterinarians of Oceanogràfic Valencia, especially Carlos Barros-García, for the obtaining and transfer of dolphin ejaculates. S.R.D. is supported by a "Doctorados Industriales 2018 " fellowship of Comunidad de Madrid (IND2018/BIO-9610).

Author Contributions: Conceptualization, L.F.C.C.; methodology, L.Q.A, R.R., M.M.F A.M.M., and A.G.-A., L.F.C.C; validation, L.Q.A, R.R., R.V., and A.G.-A., L.F.C.C.; formal analysis, L.Q.A, R.R., R.V., M.M.F., A.M.M., N.M., S.P., D.G.-P., S.R.-D., M.J.S-C., A.G.-A. and L.F.C.C.; investigation, L.Q.A, R.R., R.V., M.M.F., A.M.M., N.M., S.P., D.G.-P., S.R.-D., M.J.S-C., A.G.-A., and L.F.C.C.; resources, J.G.-A. and L.F.C.C.; data curation, L.Q.A, R.R., R.V., M.M.F., A.M.M., N.M., S.P., D.G.-P., S.R.D., M.J.S-C., A.G.-A., and L.F.C.C. writing-original draft preparation, L.Q.A., R.R., A.G.-A. and L.F.C.C. writing-review, and editing and supplementary Materials writing, L.Q.A, R.R., R.V., M.M.F., A.M.M., N.M., S.P., D.G.-P., S.R.-D., M.J.S-C., A.G.-A., and L.F.C.C; supervision, L.F.C.C. project administration, L.F.C.C. funding acquisition, A.G-A., and L.F.C.C. All authors approved the submitted manuscript. Competing interests: The authors have no competing interests to declare. Data availability: The clean RNA-seq read datasets were submitted to the Sequence Read Archive (SRA) database of NCBI, and can be consulted under the Bio project number (PRJNA703781). 


\section{Supplementary Materials for}

\section{A drastic shift in the energetic landscape of toothed whale sperm cells}

L. Q. Alves, R. Ruivo, R. Valente, M. M. Fonseca, A. M. Machado, S. Plön, N. Monteiro, D. García-Parraga, S. Ruiz-Díaz, M.L. Sánchez-Calabuig, A. GutiérrezAdán*, L. Filipe C. Castro*

Correspondence to: filipe.castro@ciimar.up.pt; agutierr@inia.es

\section{This PDF file includes:}

Materials and Methods 


\section{Materials and Methods}

Assessing the coding status of the in-study genes in Mammalia - general approach

To investigate the coding status of each target gene, the following method was employed: (i) Orthology of Cetacea genes was assessed combining phylogenetics using the Ensembl.org Gene tree pipeline and synteny analyses to authenticate one-to-one orthologues, and to define genomic regions to be collected for gene annotation; (ii) PseudoChecker (pseudochecker.ciimar.up.pt) $(12,39)$ analysis and/or manual annotation of the open reading frame (ORF) of each gene was performed over previously retrieved genomic regions, with these further being inspected for gene inactivating mutations (frameshift mutations, premature stop codons and splice site mutations); (iii) finally, for each gene and each species, at least one identified ORFabolishing mutation was further validated using independent genomic sequencing read sets obtained from the National Center of Biotechnology Information (NCBI) sequence read archive (SRA) database, when available. The sequence analysis pipeline is summarized in Fig. S2. Overall, a total of 169 mammalian genomes, including 27 Cetacea, were analyzed in the study (Table S1).

\section{Synteny analyzes}

Synteny maps were built for each of the analyzed genes in Cetacea (Figs. S3, S28, S33, S61, S68, S87, S115, S133, and S146) using NCBI-available annotated genome assemblies: Balaenoptera acutorostrata scammoni (minke whale: accession ID - GCF_000493695.1), Delphinapterus leucas (beluga whale: GCF_002288925.2), Globicephala melas (long-finned pilot whale: GCF_006547405.1), Lagenorhynchus obliquidens (Pacific white-sided dolphin: GCF_003676395.1), Lipotes vexillifer (baiji: GCF_000442215.1), Monodon monoceros (narwhal: GCF_005190385.1), Neophocaena asiaeorientalis asiaeorientalis (narrow-ridged finless porpoise: GCF_003031525.1), Orcinus orca (killer whale: GCF_000331955.1), Physeter macrocephalus (sperm whale: GCF_002837175.2) and Tursiops truncatus (bottlenose dolphin: GCF_001922835.1).

For each target gene, the Bos taurus (cow) genome was screened (GCF_002263795.1), and the corresponding ortholog gene locus was set as a reference for Cetacea. Finally, for each studied gene: $(i)$ genome assemblies with annotation of the orthologous gene, five (characterized, with available gene description or available full name) protein-coding genes, upstream and downstream of the annotated target gene were collected and placed into the syntenic map; (ii) concerning genome assemblies displaying the absence of annotation for the target gene, the corresponding $B$. taurus (cow) direct flanking genes were set as reference genes, further collecting the immediately four remaining upstream or downstream adjacent genes to these.

\section{$\underline{\text { Gene annotation }}$}

NCBI gene annotations for the mammalian orthologues of Gapdhs, Pgk2, Gk2, and Ldhc were sequence validated via PseudoChecker (pseudochecker.ciimar.up.pt), which estimates the coding status of a gene $(12,39)$. To this effect, a total of 4 PseudoChecker analyzes were run, using the human gene orthologue as a comparative coding sequence input (NCBI Accession ID regarding human Gapdhs: NM_014364.5; Pgk2: NM_138733.5; Gk2: NM_033214.3; Ldhc: NM_002301.5), as well as the 
genomic sequence encompassing the putative ORF of the orthologous gene of each target species, directly exported from the NCBI genome browser (default parameters were used, except for the minimum exon alignment identity, set to 40\%). Through PseudoIndex, a user assistant metric built into the software, we rapidly estimated the erosion condition of the tested gene on a discrete scale ranging from 0 (coding) to 5 (pseudogenized) $(12,39)$, we further classified the predicted sequences into functional (PseudoIndex between 0 and 2) or putatively pseudogenized (PseudoIndex higher than 2 ), with subsequent manual annotation and validation of eventual gene disrupting mutations (see below) (Fig. S2; Tables S2 and S5-S7).

In the case of Cetacea and Hippopotamus amphibius (hippopotamus), sequence annotation was performed on both annotated and unannotated genomes for all in-study genes (Gapdhs, Pgk2, Gk2, Ldhc, Aldoa_v2, Mpc1l, Pdha2, Oxct2a, and Oxct2b) (Fig. $\mathrm{S} 2)$. In detail, $(i)$ for genomes displaying annotation for the target genes, the genomic portions underlying the respective annotation were directly collected through the NCBI gene browser; (ii) for annotated genomes, but without the target gene annotation (e.g. Ldhc, L. obliquidens - Pacific white-sided dolphin), according to the previously produced synteny maps, the genomic sequences ranging from the upstream to the downstream flanking genes were collected and sequence analyzed; (iii) finally, for unannotated cetacean genomes (i.e., Balaenoptera bonaerensis, the Antarctic minke whale), the genomic sequences were collected via BLAST searches against the corresponding genome assembly using, as a query, a set of three sequences, including the $B$. taurus (cow) target gene corresponding ortholog coding sequence (CDS), as well as the CDS's of the flanking genes in the same species. The returned BLAST hits were analyzed, further retrieving the genomic sequence corresponding to the consensus hit across those obtained per each query sequence. For the cases where no consensual blast hit was obtained, all hits corresponding to the B. taurus (cow) CDS query were inspected, the aligning regions submitted to a back-blast search against the nucleotide (nt) database of NCBI, with the matching genomic sequence(s) corresponding to the gene of interest being the one(s) selected for annotation (when existing) (Table S8).

The collected genomic sequences harboring the putative ORF of the in-study genes were imported into Geneious Prime 2020 software (www.geneious.com) (40) and the gene CDS was manually annotated using, as a reference, the B. taurus (cow) corresponding orthologs concerning cetaceans and H. amphibius (hippopotamus) and, for non-cetacean mammals, the H. sapiens (human) counterparts, except for Aldoa_v2 which made use of the Mus musculus (mouse) reference ortholog (Table S8). Explicitly, per gene and species, by using the Geneious (40) built-in map to reference tool, and with the highest sensitivity parameter selected, each (3' and 5' untranslated region-flanked) reference species' coding-exon (in the cases of Gk2, Pgk2, Mpcll, $P d h a 2, O x c t 2 a$, and $O x c t 2 b$ genes, the corresponding reference single coding-exon) was mapped against the target genomic sequence. Due to alignment issues regarding the exon 2 of B. taurus (cow) Gapdhs against Cetacea, this was first mapped against the corresponding $H$. amphibius (hippopotamus) genomic sequence, further using the predicted exon as the reference Gapdhs exon 2 for annotation in cetaceans. Regarding $O x c t 2 a$ and $O x c t 2 b$ gene annotations, to ensure that we were not mistakenly annotating 
each of these for the corresponding copy, we first mapped the exons of the respective $B$. taurus (cow) flanking genes, forcing the cow ortholog of the Oxct2 copy of interest to align within the corresponding flanked genomic sequence (Pabpc4 - XM_010803664.2 and Macf1 - NM_001143860.2 for Oxct2a; Trit1 - NM_001192840.1 and Ppie NM_001098161.1 for $O x c t 2 b$ ). The cases for which we could not map at least one of each copy's flanking genes were rendered inconclusive. Finally, each exon alignment was further screened for gene disruptive mutations, including in-frame premature stop codons, frameshift, and splice site mutations (any deviation from the consensus donor splice site GT/GC or the consensus acceptor splice site AG) (Figs. S4, S29, S34, S62, S69, S88, S116, S134, and S147).

Validation of gene erosion lesions

To ensure that the identified genetic lesions were not the result of sequencing and/or genome assembly artifacts, we further validated at least one of these via the mapping of unassembled genomic sequencing reads retrieved from two independent genomic projects from the NCBI sequence read archive, when available. To accomplish this, blastn searches were conducted against the selected SRA projects, using as a query the nucleotide sequence containing the selected mutation(s). The matching sequencing reads were imported into Geneious Prime 2020 (40) software and mapped against the manually annotated mutation using the built-in map to reference tool (highest sensibility parameter selected), further confirming, or not, the presence of the identified mutation (Figs. S5-S21, S23-S27, S30-S32, S35-S60, S63-S67, S70-S86, S89-114, S117-132, S135-145, and S148-158).

Assessing the Aldoa somatic and testis-specific isoforms expression status in other $\underline{\text { mammals }}$

To confirm the testis-restrictive expression of the Aldoa_v2 isoform in $H$. sapiens (human), B. taurus (cow), and M. musculus (mouse), we inspected the transcriptomes of four different tissues in each of these species, including lung, kidney, skin, and testis. To accomplish this: $(i)$ for each species, we have directly imported the genomic portion corresponding to the Aldoa gene using the NCBI genome browser into Geneious (40) (human corresponding genomic accession ID: NC_000016.10; cow: NC_037352.1; mouse: NC_000073.7) and, with the built-in map to reference tool (high sensitivity parameter selected), we annotated both isoforms using the mouse ortholog as a reference (Aldoa_vl accession ID: NM_007438.4; Aldoa_v2: NM_001177307.1); (ii) per tissue and species, we inspected two independent NCBI SRA transcriptomic projects or samples, and run a blastn search against each of them using, as reference, the respective CDS of both Aldoa_v1 and v2 isoforms (-max_target_seqs 5000) (Table S9); (iii) the corresponding reads were imported into Geneious (40) and these were further mapped against each species respective isoform annotations (maximum read gap size adjusted to the length of each isoform with a maximum mismatch rate of $2 \%$ ); (iv) finally, aligning reads were count per species, tissue and isoform, with isoforms displaying at least 30 aligning reads being considered as expressed (Table S9). 


\section{Selection analysis and RELAX}

To determine the direction of natural selection (relaxed or intensified) in Gapdhs, Pgk2, Gk2, and Ldhc genes across the mammalian tree, we used the RELAX software on the Hyphy package $(41,42)$. For this purpose, in mammal species displaying ORF-abolishing mutations in the coding region of these target genes, we manually recovered the gene sequence, removed frameshift insertions and in-frame stop codons were recorded as missing data (NNN). For species for which no evidence of disruption was found during the PseudoChecker analyses, we directly collected the respective CDSs from the NCBI database. Excluding cetaceans, we considered a species as having the target gene mutated only for the cases where we could validate ORF-disruptive mutations using data from at least 1 SRA genomic project (mandatorily different from the reference genome corresponding project) or 2 distinct individuals. Mammals displaying issues related to exon alignment and fragmented genomic regions in the target gene and/or exon deletions were excluded from the analysis. In Tables S10, $\mathrm{S} 11, \mathrm{~S} 12$, and S13, we present the list of mammals used in RELAX analysis for each target gene.

For each gene, mammalian sequences were translated-aligned in Geneious Prime 2020 (40) using the Blosum62 substitution matrix. RELAX estimates a relaxation/intensification parameter $\mathrm{k}$ that either indicates relaxed selection $(\mathrm{k}<1)$ or intensified selection $(\mathrm{k}>1)$. Using previous alignments as input, we ran the test by specifying ( $i$ ) different cetacean families as foreground branches; (ii) each mammalian clade (or species) presenting ORF-disrupting mutations as test set; (iii) different mammalian orders whose number of species used in this analysis was higher than 10 . For all the tests, all the other branches leading to species that did not lose the target gene were specified as background. Specifically, for Gapdhs, given the consensual loss pattern observed in Odontoceti, we ran RELAX considering (in contrast to other genes) (i) all Odontoceti species as foreground branches; (ii) all Mysticeti species as the test set; (iii) and all mammalian orders except Marsupial orders. We report all values of $\mathrm{k}$ and three omega classes, together with the percentage of sites under these $\mathrm{dN} / \mathrm{dS}$ rates for each run in Table S3.

Mitochondrial size measurements across Mammalia

To further examine the mitochondrial size of mammalian sperm, we collected published photographs of longitudinal sections through the midpiece of the sperm flagellum (43-60). For well-defined mitochondrion, for which we were able to see its boundaries, we took two measurements: one called "Height" defined as the measurement of the mitochondrion parallel to the axoneme, and another called "Width" defined as the measurement of the mitochondrion perpendicular to the axoneme (Table S14). Images for which only magnification was displayed, the height and width of each mitochondrion were calculated from distances measured with a ruler $( \pm 0.05 \mathrm{~cm})$. On the other hand, for images displaying scale, we used the ImageJ software (61) to automatically calculate both measures. For the majority of the species, data on mitochondrial size was only taken from one individual/figure (Table S14).

Finally, a scatterplot was constructed using R software (62), allowing the comparison between mitochondrial sizes in different mammalian lineages (Fig. S159). 
As both measurements were not normally distributed, we tested for differences between mammalian orders using the nonparametric Kruskall-Wallis test. Moreover, for each measurement, each order was compared to all the others (i.e., base-mean) to determine if the height/width of their mitochondria was, significantly bigger or smaller. Boxplots were developed allowing for a visual comparison of the differences in the distribution of data across several mammalian orders (Fig. S160).

\section{Ethics of experimentation}

Dolphin spermatozoa were collected from two adult bottlenose dolphins ( $T$. truncatus) as part of the routine husbandry training methods for the bottlenose dolphin on a voluntary basis at Oceanogràfic-Valencia (Valencia, Spain). All procedures were performed using methods consistent with the Animal Care Protocol of the Oceanogràfic-Valencia. All animals used in this project were cared for using procedures that are consistent with the regulations and policies of Oceanogràfic-Valencia and the Spanish Council on Animal Care Documents, 'Guide to the care and Use of Experimental Animals', 'Categories of Invasiveness in Animal Experiments' and 'Ethics of Animal Experimentation'.

\section{Semen collection and cryopreservation}

Semen collection has been previously described (63). After collection, semen samples (avoiding contact with any source of light) were diluted at a 1:1 ratio using a commercial extender [Beltsville Thawing Solution (BTS)] (GVP, Zoitech Lab, Madrid, Spain) and frozen in TRIS egg yolk-based buffer containing glycerol to obtain a 3\% final glycerol concentration and a final concentration of $200 \times 106 \mathrm{sperm} / \mathrm{mL}$ (63).

\section{Dolphin sperm RNA extraction, construction, processing, and analysis}

The total RNA content was extracted using one sample of Dolphin sperm as follows. Semen samples were washed with sperm preparation medium (CooperSurgical, Denmark) and gently centrifuged at $4^{\circ} \mathrm{C}$. Cell pellets were ressuspended in NZYol reagent (Nzytech, Portugal) and incubated at $65^{\circ} \mathrm{C}$ for 5 minutes. Following extraction procedure and done in according to the manufacturer's instructions in combination with the Illustra RNAspin Mini RNA Isolation Kit (GE Healthcare, UK) with on-column DNase I digestion and elution in RNase-free water. The total RNA concentration was measured in a microplate spectrophotometer with Take ${ }^{\mathrm{TM}}$ Micro-Volume Plate (BioTeK, USA) and its quality was checked through the measurement of the OD260/280 ratio values (1.8-2.0). The RNA integrity was verified by running $1 \mu 1$ in a $1 \%$ agarose gel. The sample was shipped to Macrogen (Seoul, Korea), the strandspecific library was built (Insert size: $250-300 \mathrm{bp}$ ) and the sequencing was performed with an Illumina NovaSeq 6000 platform (Paired-end 150 bp).

The RNA-Seq dataset was processed with the protocol and parameters applied in Machado et al. (2020) (64). Briefly, the FastQC (65) (0.11.8) software was used to check the initial quality of the dataset, Trimmomatic (66) (0.38) to trim and qualityfilter the raw reads, Rcorrector (67) (1.0.3) to correct the sequencing errors, and Centrifuge (68) (1.0.3-beta) to taxonomically classify and filter out exogenous raw reads and possible sources of contamination. All reads corresponding to match hits out of the Mammalia class (Taxon ID: 40674) were removed. To complement the dataset 
assessment, the clean reads were mapped onto the NCBI-available genome assembly of T. truncatus (bottlenose dolphin) (GCF_001922835.1) resorting to Hisat2 (69) (2.2.0) (default settings), calculating the rate of mapped reads with samtools (70) (1.9.0). The stats of raw and clean datasets, as well as the rate mapping against the reference, can be consulted in Table S15. The clean datasets were submitted to the NCBI SRA database and can be consulted under the BioProject - PRJNA703781.

Finally, resorting to the produced transcriptomic dataset, we inspected the occurrence of expression reads from Gapdhs, Gk2, and 5 highly testis-expressed genes according to the Human Protein Atlas (www.proteinatlas.org): Ybxl, Phf7, Tsacc, Tnpl, and Eeflg. To accomplish this: $(i)$ we collected the T. truncatus (bottlenose dolphin) CDS of the previously annotated Gapdhs, Gk2, as well as the control genes respective CDS from NCBI, and ran a blast search of each of them against the clean reads dataset (-task megablast, -max_target_seqs 20000); (ii) the reads corresponding to each blast search were collected and mapped against the respective gene using the Geneious (40) built-in map to reference tool (maximum read gap size adjusted to the length of each gene with a maximum mismatch rate of 2\%); (iii) the splicing patterns were inspected and the aligning regions covering mutated regions screened for mutated transcripts (Fig. S22). A similar approach was employed to deduce the presence of mRNA of these genes (here, also including $P g k 2$ ) in mouse sperm cells (NCBI SRA - BioProject PRJNA320896).

\section{Effect of glucose and pyruvate on sperm motility}

Sperm starvation was done in a HEPES modified Toyoda-Yokoyama-Hosi medium (HEPES-TYH) (71) according to changes in the composition that have been previously indicated (23), for which glucose and pyruvate were omitted. This medium can be supplemented with $\mathrm{HCO}^{-}$or BSA to support capacitation. Thawing was done in a water bath at $37^{\circ} \mathrm{C}$ for $50 \mathrm{~s}$. Then spermatozoa were separated on a density gradient BoviPure $^{\mathrm{TM}}$ (Nidacon, Sweden) and resuspended in HEPES-TYH medium, and then washed by centrifugation for $5 \mathrm{~min}$ at $300 \mathrm{~g}$, and resuspended in $1 \mathrm{ml}$ of HEPES-TYH medium. Sperm were incubated with or without glucose and pyruvate at $5 \% \mathrm{CO} 2$ at $38^{\circ} \mathrm{C}$ in TYH medium supplemented with $15 \mathrm{mM} \mathrm{HCO}^{-}$and $5 \mathrm{mg} / \mathrm{mL}$ bovine serum albumin (BSA, fatty acid-free, A0281) for 3 days. Motility and kinetic were reported every day by the Integrated Semen Analysis System (ISAS; Projectes i ServeisR + D S.L., Valencia, Spain) and acrosome status and vitality were analyzed.

\section{Fatty acid oxidation inhibition}

To ascertain what energy source is used by dolphin spermatozoa, we incubated sperm samples with etomoxir, a selective inhibitor of CPT1, the rate-limiting enzyme of b-oxidation. Sperm samples from the two adult dolphins $(n=6$, triplicate experiment) were incubated in HEPES-TYH supplemented with $3 \%(\mathrm{w} / \mathrm{v})$ fatty-acid-free bovine serum albumin and $1 \%(\mathrm{v} / \mathrm{v})$ penicillin-streptomycin-neomycin at $38{ }^{\circ} \mathrm{C}, 5 \% \mathrm{CO} 2$, in the presence or absence (control) of $1 \mathrm{mM}$ of etomoxir. Motility and kinetic (ISAS analysis), and acrosome status were reported after $1 \mathrm{~h}, 2 \mathrm{~h}$, and $18 \mathrm{~h}$. 
Sperm motility and kinetics

Sperm motility was objectively determined using ISAS. Ten microliters of sperm suspension were placed in a Mackler chamber on the stage heated to $37^{\circ} \mathrm{C}$ of a Nikon Eclipse E400 (Nikon, Tokyo, Japan) fitted with a digital camera, Basler acA1300-200uc (Basler AG, Ahrensburg, Germany). Three to five movies of $1.5 \mathrm{~s}$ were recorded at $60 \mathrm{frames} / \mathrm{s}$ using the software Pylon Viewer provided by Basler, capturing at least 100 moving spermatozoa (72). The motility and sperm kinetics were analyzed using the free software ImageJ (61) with the plugin ISAS bmg following instructions for analyzing dolphin spermatozoa (73). The parameters analyzed were as described by Mortimer et al. (74): straight-line velocity (VSL; $\mu \mathrm{m} / \mathrm{s}$ ), curvilinear velocity (VCL; $\mu \mathrm{m} / \mathrm{s})$, average path velocity (VAP; $\mu \mathrm{m} / \mathrm{s})$, linearity (LIN) (defined as (VSL/VCL) $\times$ $100)$, straightness (STR) (defined as (VSL/ VAP) $\times 100$ ), wobble (WOB) (defined as $(\mathrm{VAP} / \mathrm{VCL}) \times 100)$, amplitude of lateral head $(\mathrm{ALH})$ displacement $(\mu \mathrm{m})$, and beat-cross frequency (BCF; Hz). Also, we examined the percentage of spermatozoa showing more signs of hyperactivation (HYP) by determining out of all the analyzed spermatozoa (9340) the lower VCL and ALH values of the 10\% of spermatozoa with the highest $\mathrm{VCL}$ and ALH. These values were: $\mathrm{VCL}=150 \mu \mathrm{m} / \mathrm{s}$ and $\mathrm{ALH}=5.5 \mu \mathrm{m}$. Thus, we defined spermatozoa showing hyperactive-like motility as those showing VCL $>150$ $\mu \mathrm{m} / \mathrm{s}$ and ALH $>5.5 \mu \mathrm{m}(75)$.

\section{Sperm preparation and purification}

Semen samples from 2 dolphins (T. truncatus) and 3 Friesian bulls (B. taurus) were analyzed. Frozen sperm from 2 adult bottlenose dolphins spermatozoa were separated as previously indicated on a density gradient BoviPure ${ }^{\text {TM }}$ (Nidacon, Sweden) and resuspended in PBS medium, and then washed two times by centrifugation for 5 $\mathrm{min}$ at $300 \mathrm{~g}$. Frozen semen straws $(0.25 \mathrm{~mL})$ from 3 Friesian bulls previously tested for IVF were thawed at $37^{\circ} \mathrm{C}$ in a water bath for $1 \mathrm{~min}$ and centrifuged for $10 \mathrm{~min}$ at $300 \mathrm{~g}$ through a gradient of $1 \mathrm{~mL}$ of $40 \%$ and $1 \mathrm{~mL}$ of $80 \%$ Bovipure (Nidacon Laboratories $\mathrm{AB}$, Göthenborg, Sweden), according to the manufacturer's instructions. The sperm pellet was isolated and washed two times in PBS by centrifugation at $300 \mathrm{~g}$ for $5 \mathrm{~min}$. For all the samples, after the last centrifugation cells were frozen with liquid nitrogen and stored at $-80^{\circ} \mathrm{C}$ for further metabolite extraction.

\section{Metabolite extraction for by proton nuclear magnetic resonance spectroscopy $\left({ }^{1} \mathrm{H}\right.$ $\underline{\text { NMR) }}$}

A significant number of cells ( 200 million) were used to extract enough metabolites for each ${ }^{1} \mathrm{H}$ NMR spectroscopy experiment. Two different pools of samples with identical phenotypes were prepared: 3 bulls and 2 dolphins. Frozen seminal samples from three different Asturian Valley bulls (B. taurus) were used. Bulls were housed at the Cenero AI Centre [Regional Service of Agrifood Research and Development (SERIDA), Gijón, Spain], complying with all European Union regulations for animal husbandry. Additional approval from an ethical committee to conduct this study was not required. Animals were selected based on being good breeders by having AI outcomes using frozen samples. Sperm from the two dolphins used in the motility experiments were used. Thawed semen was washed by density gradient centrifugation by placing it on top of a BoviPure gradient (Nidacon 
Laboratories AB, Göthenborg, Sweden) of $1 \mathrm{~mL}$ at $80 \%$ (lower layer) and $1 \mathrm{~mL}$ at $40 \%$ (upper layer) and centrifuging for $5 \mathrm{~min}$ at 300xg. Sperm cells were then washed three times with cold PBS and cell pellets were frozen with liquid nitrogen and stored at -80 ${ }^{\circ} \mathrm{C}$ for further metabolite extraction. Extraction of low-molecular-weight metabolites was performed following a previously published procedure (76). For the preparation of the ${ }^{1} \mathrm{H}$ NMR samples, semen cell pellets were pre-treated. A methanol extraction was performed with the following protocol: samples were defrosted to room temperature for 5 minutes slowly within the ice. $1.3 \mathrm{~mL}$ of $\mathrm{CHCl} 3: \mathrm{MeOH}: \mathrm{ddH} 2 \mathrm{O}$ in a ratio 41.7:35.6:32.7 (v/v/v) was added to the sperm sample. The Eppendorf with the extraction was placed at $4{ }^{\circ} \mathrm{C}$ with agitation for $4 \mathrm{~h}$. The mixture was centrifuged at $4{ }^{\circ} \mathrm{C}$ at max-speed ( 25000-30000 g) for 30 mins. The upper phase of the dissolution (Hydrophilic phase) was transferred to a new $2 \mathrm{~mL}$ Eppendorf. The down phase of the dissolution (Lipophilic phase) was transferred to a different Eppendorf too. All the samples were then dried in a Speed-Vac. For the preparation of the hydrophilic samples to be acquired in the ${ }^{1} \mathrm{H}$ NMR we have resuspended the lyophilized product with 500 $\mu \mathrm{L}$ of deuterium oxide (D2O) and $0.11 \mu \mathrm{M}$ of DSS (Sodium trimethylsilylpropanesulfonate). Samples were briefly vortexed and $500 \mu \mathrm{L}$ of the semen extract was finally pipetted into a $5 \mathrm{~mm}{ }^{1} \mathrm{H}$ NMR tube. Lipophilic extracts were resuspended in $300 \mu \mathrm{l}$ of DMSO-d6 with $4 \mathrm{mM}$ of TPP (triphenylphosphine) and transferred to a Shigemi tube. In all cases, sample preparation was manually done at $298 \mathrm{~K}$.

\section{${ }^{1} \mathrm{H}$ NMR measurements}

All ${ }^{1} \mathrm{H}$ NMR experiments were recorded at $298 \mathrm{~K}$ on a Bruker $600 \mathrm{MHz}(12 \mathrm{~T})$ Avance III spectrometer equipped with a $\mathrm{BBO}(\mathrm{BB}, 1 \mathrm{H})$ probe head. For each sample, a 1D $1 \mathrm{H}$ p3919gp with water signals suppression using a binomial 3-9-19 pulse with echo gradient pair (21 min). Data analysis was done using the TopSpin 3.5 software (Bruker Biospin $\mathrm{GmbH}$ ) (77). Free induction decays were multiplied by an exponential function equivalent to $0.3 \mathrm{~Hz}$ line-broadening before applying Fourier transform. All transformed spectra were corrected for phase and baseline distortions and referenced to the DSS singlet at $0 \mathrm{ppm}$. The hydrophilic and lipophilic samples were recorded.

The identification of the metabolites was done on the sample with the higher concentration of sperm extracts. DSS was used as an internal reference that can be used to quantify the number of different molecules. TSP shows a peak at $0 \mathrm{ppm}$, which corresponds to 9 equivalent protons. Therefore, this peak is equivalent to a $1 \mathrm{mM}$ proton intensity. The peaks are then integrated and referenced to DSS. On the other hand, $4 \mathrm{mM}$ of TPP was added to the lipophilic phase. Considering the contribution of $15 \mathrm{H}$ the peak integral corresponds to $60 \mathrm{mM}$. However, the quantification of the lipophilic phase is impossible due to the different contributions coming from the sample type of compound. For example, a signal coming phosphatidylcholine will be composed of the contribution of the - $\mathrm{CH} 2$ - groups in their hydrophobic tail, and the length of the tail will vary randomly. Thus, the integrals can be compared as relative integrals, not absolute quantification. 
$\underline{\text { Statistical analysis }}$

Statistical analysis was carried out using the software package GraphPad Prism 8.0.2 for Windows (GraphPad Software, San Diego, CA, USA). Results are expressed as means \pm standard error of the mean (SEM). Means were compared and analyzed using a one-tailed paired-sample Student's t-test or repeated measures one-way analysis of variance (ANOVA), followed by Tukey's post hoc test. Significance was set at $\mathrm{p}<$ 0.05 . 